\title{
Some Generalized Difference Sequence Spaces Defined by a Sequence of Moduli in $n$-Normed Spaces
}

\author{
Abdullah Alotaibi, ${ }^{1}$ Kuldip Raj, ${ }^{2}$ and S. A. Mohiuddine ${ }^{1}$ \\ ${ }^{1}$ Operator Theory and Applications Research Group, Department of Mathematics, Faculty of Science, King Abdulaziz University, \\ P.O. Box 80203, Jeddah 21589, Saudi Arabia \\ ${ }^{2}$ School of Mathematics, Shri Mata Vaishno Devi University, Katra, Jammu and Kashmir 182320, India
}

Correspondence should be addressed to S. A. Mohiuddine; mohiuddine@gmail.com

Received 12 December 2014; Revised 17 February 2015; Accepted 25 February 2015

Academic Editor: Mohamed-Aziz Taoudi

Copyright (C) 2015 Abdullah Alotaibi et al. This is an open access article distributed under the Creative Commons Attribution License, which permits unrestricted use, distribution, and reproduction in any medium, provided the original work is properly cited.

We introduce some new generalized difference sequence spaces by means of ideal convergence, infinite matrix, and a sequence of modulus functions over $n$-normed spaces. We also make an effort to study several properties relevant to topological, algebraic, and inclusion relations between these spaces.

\section{Introduction and Preliminaries}

The concept of 2-normed spaces was initially developed by Gähler [1] in the middle of 1960s, while that of $n$-normed spaces one can see in Misiak [2]. Since then, many others have studied this concept and obtained various results; see Gunawan [3, 4] and Gunawan and Mashadi [5]. Let $n \in \mathbb{N}$ and $X$ be a linear space over the field $\mathbb{R}$ of reals of dimension $d$, where $d \geq n \geq 2$. A real valued function $\|\cdot, \ldots, \cdot\|$ on $X^{n}$ satisfying the following four conditions:

(1) $\left\|x_{1}, x_{2}, \ldots, x_{n}\right\|=0$ if and only if $x_{1}, x_{2}, \ldots, x_{n}$ are linearly dependent in $X$,

(2) $\left\|x_{1}, x_{2}, \ldots, x_{n}\right\|$ is invariant under permutation,

(3) $\left\|\alpha x_{1}, x_{2}, \ldots, x_{n}\right\|=|\alpha|\left\|x_{1}, x_{2}, \ldots, x_{n}\right\|$ for any $\alpha \in \mathbb{R}$,

(4) $\left\|x+x^{\prime}, x_{2}, \ldots, x_{n}\right\| \leq\left\|x, x_{2}, \ldots, x_{n}\right\|+\left\|x^{\prime}, x_{2}, \ldots, x_{n}\right\|$,

is called a $n$-norm on $X$ and the pair $(X,\|\cdot, \ldots, \cdot\|)$ is called a $n$-normed space over the field $\mathbb{R}$. For more details about $n$ normed spaces, see $[6,7]$ and references therein.

The notion of difference sequence spaces was introduced by Kizmaz [8], who studied the difference sequence spaces $l_{\infty}(\Delta), c(\Delta)$, and $c_{0}(\Delta)$. The notion was further generalized by Et and Çolak [9] by introducing the spaces $l_{\infty}\left(\Delta^{m}\right), c\left(\Delta^{m}\right)$, and $c_{0}\left(\Delta^{m}\right)$. Later the concept has been studied by Bektaş et al. [10] and Et and Esi [11]. Another type of generalization of the difference sequence spaces is due to Tripathy and Esi [12] who studied the spaces $l_{\infty}\left(\Delta_{v}\right), c\left(\Delta_{v}\right)$, and $c_{0}\left(\Delta_{v}\right)$. Recently, Esi et al. [13] and Tripathy et al. [14] have introduced a new type of generalized difference operators and unified those as follows.

Let $m$ and $v$ be nonnegative integers; then for $Z$, a given sequence space, we have

$$
Z\left(\Delta_{v}^{m}\right)=\left\{x=\left(x_{k}\right) \in w:\left(\Delta_{v}^{m} x_{k}\right) \in Z\right\},
$$

for $Z=c, c_{0}$, and $l_{\infty}$ where $\Delta_{v}^{m} x=\left(\Delta_{v}^{m} x_{k}\right)=\left(\Delta_{v}^{m-1} x_{k}-\right.$ $\left.\Delta_{v}^{m-1} x_{k+v}\right)$ and $\Delta_{v}^{0} x_{k}=x_{k}$, for all $k \in \mathbb{N}$, which is equivalent to the following binomial representation:

$$
\Delta_{v}^{m} x_{k}=\sum_{\nu=0}^{m}(-1)^{v}\left(\begin{array}{l}
m \\
v
\end{array}\right) x_{k+v v} .
$$

Taking $v=1$, we get the spaces $l_{\infty}\left(\Delta^{m}\right), c\left(\Delta^{m}\right)$, and $c_{0}\left(\Delta^{m}\right)$ studied by Et and Çolak [9]. Taking $v=m=1$, we get the spaces $l_{\infty}(\Delta), c(\Delta)$, and $c_{0}(\Delta)$ introduced and studied by Kizmaz [8]. For more details about difference sequence spaces, see [15-19].

A modulus function is a function $f:[0, \infty) \rightarrow[0, \infty)$ such that

(1) $f(x)=0$ if and only if $x=0$,

(2) $f(x+y) \leq f(x)+f(y)$, for all $x, y \geq 0$, 
(3) $f$ is increasing,

(4) $f$ is continuous from the right at 0 .

It follows that $f$ must be continuous everywhere on $[0, \infty)$. The modulus function may be bounded or unbounded. For example, if we take $f(x)=x /(x+1)$, then $f(x)$ is bounded. If $f(x)=x^{p}, 0<p<1$, then the modulus function $f(x)$ is unbounded. Subsequently, modulus function has been discussed in [20] and references therein.

In [21], Mursaleen introduced the idea of $\lambda$-statistical convergence by extending the concept of $[V, \lambda]$ summability. Further, Savaş and Das [22] unified the two approaches and gave new concepts of $I$-statistical convergence, $I-S_{\lambda^{-}}$ convergence, and $I-[V, \lambda]$ convergence. Later some pioneer works have been extended in this direction by numerous authors such as Belen and Mohiuddine [23], Das et al. [24], Gürdal and Sarí [25], and references therein. Quite recently, many authors including Et et al. [26] and Maddox [27] have constructed some sequence spaces by using modulus function and difference sequences and investigate their properties.

Let $X$ and $Y$ be two sequence spaces and $A=\left(a_{n k}\right)$ be an infinite matrix of real or complex numbers $a_{n k}$, where $n, k \in$ $\mathbb{N}$. Then we say that $A$ defines a matrix mapping from $X$ into $Y$ if, for every sequence $x=\left(x_{k}\right)_{k=0}^{\infty} \in X$, the sequence $A x=$ $\left\{A_{n}(x)\right\}_{n=0}^{\infty}$, the $A$-transform of $x$, is in $Y$, where

$$
A_{n}(x)=\sum_{k=0}^{\infty} a_{n k} x_{k} \quad(n \in \mathbb{N}) .
$$

By $(X, Y)$, we denote the class of all matrices $A$ such that $A$ : $X \rightarrow Y$. Thus, $A \in(X, Y)$ if and only if the series on the righthand side of (3) converges for each $n \in \mathbb{N}$ and every $x \in X$.

The matrix domain $X_{A}$ of an infinite matrix $A$ in a sequence space $X$ is defined by

$$
X_{A}=\left\{x=\left(x_{k}\right): A x \in X\right\} .
$$

The approach constructing a new sequence space by means of the matrix domain of a particular limitation method has recently been employed by several authors (see [28]).

Let $\lambda=\left(\lambda_{n}\right)$ be a nondecreasing sequence of positive numbers such that $\lambda_{n+1} \leq \lambda_{n}+1, \lambda_{1}=1, \lambda_{n} \rightarrow \infty$ as $n \rightarrow \infty$, and $I_{n}=\left[n-\lambda_{n}+1, n\right]$.

Let $\mathbb{N}$ be a nonempty set. Then a family of sets $I \subseteq 2^{\mathbb{N}}$ (power set of $\mathbb{N}$ ) is said to be an ideal if $I$ is additive; that is, $A, B \in I \Rightarrow A \cup B \in I$ and $A \in I, B \subseteq A \Rightarrow B \in I$. A nonempty family of sets $E(I) \subseteq 2^{\mathbb{N}}$ is said to be filtered on $\mathbb{N}$ if and only if $\Phi \notin \mathfrak{E}(I)$; for $A, B \in \mathfrak{E}(I)$ we have $A \cap B \in \mathfrak{E}(I)$ and for each $A \in \mathfrak{E}(I)$ and $A \subseteq B$ implying $B \in \mathfrak{E}(I)$.

An ideal $I \subseteq 2^{\mathbb{N}}$ is called nontrivial if $I \neq 2^{\mathbb{N}}$. A nontrivial ideal $I \subseteq 2^{\mathbb{N}}$ is called admissible if $\{\{x\}: x \in \mathbb{N}\} \subseteq I$. A nontrivial ideal is maximal if there cannot exist any nontrivial ideal $J \neq I$ containing $I$ as a subset. For each ideal $I$, there exist a filter $\mathfrak{E}(I)$ corresponding to $I$; that is, $\mathfrak{E}(I)=\{K \subseteq \mathbb{N}$ : $\left.K^{c} \in I\right\}$, where $K^{c}=\mathbb{N} \backslash K$.

Definition 1. A sequence $x=\left(x_{k}\right)$ in a $n$-normed space $(X,\|\cdot, \ldots, \cdot\|)$ is said to be statistically convergent to some $L \in X$ if, for each $\varepsilon>0$, the set $A(\varepsilon)=\left\{k \in \mathbb{N}: \| x_{k}-\right.$ $\left.L, z_{1}, \ldots, z_{n-1} \| \geq \varepsilon\right\}$ has its natural density zero.
Definition 2 (see [26]). A sequence $x=\left(x_{k}\right)$ is said to be $\lambda_{X^{-}}^{m}$ statistically convergent to the number $L$, if, for every $\varepsilon>0$, $\lim _{n \rightarrow \infty}\left(1 / \lambda_{n}\right)\left|\left\{k \in I_{n}:\left\|\Delta^{m} x_{k}-L\right\| \geq \varepsilon\right\}\right|=0$. In this case one writes $S_{\lambda}\left(\Delta^{m}, X\right)-\lim _{k \rightarrow \infty} x_{k}=L$.

Definition 3 (see [22]). A sequence $x=\left(x_{k}\right)$ is said to be $I-[V, \lambda]$ summable to $L$, if, for any $\delta>0,\{n \in \mathbb{N}$ : $\left.\left(1 / \lambda_{n}\right) \sum_{k \in I_{n}}\left\|x_{k}-L\right\| \geq \delta\right\} \in I$, where $I_{n}=\left[n-\lambda_{n}+1, n\right]$.

Definition 4 (see [22]). A sequence $x=\left(x_{k}\right)$ is said to be $I-\lambda$ statistically convergent or $I-S_{\lambda}$ convergent to $L$ if, for every $\varepsilon>0$ and $\delta>0,\left\{n \in \mathbb{N}:\left(1 / \lambda_{n}\right)\left|\left\{k \in I_{n}:\left\|x_{k}-L\right\| \geq \delta\right\}\right| \in I\right.$. In this case one writes $x_{k} \rightarrow L\left(I-S_{\lambda}\right)$ or $I-S_{\lambda}-\lim _{k \rightarrow \infty} x_{k}=L$.

The following well-known lemma is required for establishing some important results in this paper.

Lemma 5. Let $\mathscr{F}=\left(f_{k}\right)$ be a sequence of modulus functions and $0<\delta<1$. Then for each $x>\delta$ one has $f_{k}(x) \leq 2 f_{k}(1) x / \delta$.

Throughout the paper, $\mathbb{N}$ will denote the set of all positive integers. By $S_{X}^{n}$ we denote the space of all sequences defined over $n$-normed space $(X,\|\cdot, \ldots, \cdot\|)$.

Let $I \subseteq 2^{\mathbb{N}}$ be an admissible ideal, $\mathscr{F}=\left(f_{k}\right)$ a sequence modulus functions, $p=\left(p_{k}\right)$ a bounded sequence of positive (strictly) real numbers, $u=\left(u_{k}\right)$ a sequence of positive real numbers, $A=\left(a_{n k}\right)$ an infinite matrix, and $(X,\|\cdot, \ldots, \cdot\|)$ a $n$ normed space. Then, for every $\delta>0$ and $z_{1}, \ldots, z_{n-1} \in X$, we define the following sequence spaces:

$$
\begin{aligned}
& V_{\mathscr{F}}^{I}\left[\Delta_{v}^{m}, \lambda, A, u, p,\|\cdot, \ldots, \cdot\|\right]_{0} \\
& =\left\{x=\left(x_{k}\right) \in S_{X}^{n}\right. \text { : } \\
& \{n \in \mathbb{N}: \\
& \left.\left.\frac{1}{\lambda_{n}} \sum_{k \in I_{n}} a_{n k}\left[f_{k}\left(\left\|u_{k} \Delta_{v}^{m} x_{k}, z_{1}, \ldots, z_{n-1}\right\|\right)\right]^{p_{k}} \geq \delta\right\} \in I\right\}, \\
& V_{\mathscr{F}}^{I}\left[\Delta_{v}^{m}, \lambda, A, u, p,\|\cdot, \ldots, \cdot\|\right] \\
& =\left\{x=\left(x_{k}\right) \in S_{X}^{n}:\right. \\
& \{n \in \mathbb{N}: \\
& \left.\frac{1}{\lambda_{n}} \sum_{k \in I_{n}} a_{n k}\left[f_{k}\left(\left\|u_{k} \Delta_{v}^{m} x_{k}-L, z_{1}, \ldots, z_{n-1}\right\|\right)\right]^{p_{k}} \geq \delta\right\} \\
& \in I\}
\end{aligned}
$$


for some $L>0$, and

$$
\begin{aligned}
& V_{\mathscr{F}}^{I}\left[\Delta_{v}^{m}, \lambda, A, u, p,\|\cdot, \ldots, \cdot\|\right]_{\infty} \\
& =\left\{\begin{array}{l}
x=\left(x_{k}\right) \in S_{X}^{n}: \\
\{n \in \mathbb{N}: \\
\\
\quad \frac{1}{\lambda_{n}} \sum_{k \in I_{n}} a_{n k}\left[f_{k}\left(\left\|u_{k} \Delta_{v}^{m} x_{k}-L, z_{1}, \ldots, z_{n-1}\right\|\right)\right]^{p_{k}} \geq K
\end{array}\right\} \\
& \in I\},
\end{aligned}
$$

for some $K>0$.

If we take $\mathscr{F}(x)=f_{k}(x)=x, u=\left(u_{k}\right)=1$ for all $k \in \mathbb{N}$, $v=1$, and $A=\left(a_{n k}\right)=I$ (identity matrix) and $n$-normed space is replaced by 2 -normed space then we get the sequence spaces defined by Kumar et al. [29].

The following inequality will be used throughout the paper. Let $p=\left(p_{k}\right)$ be a sequence of positive real numbers with $0<p_{k} \leq \sup _{k} p_{k}=H$, and let $D=\max \left\{1,2^{H-1}\right\}$. Then, for the factorable sequences $\left(a_{k}\right)$ and $\left(b_{k}\right)$ in the complex plane, we have

$$
\left|a_{k}+b_{k}\right|^{p_{k}} \leq D\left(\left|a_{k}\right|^{p_{k}}+\left|b_{k}\right|^{p_{k}}\right)
$$

\section{Main Results}

The main purpose of this section is to study some topological properties and some inclusion relations between the sequence spaces which we have defined above.

Theorem 6. Suppose $\mathscr{F}=\left(f_{k}\right)$ is a sequence of modulus functions, $p=\left(p_{k}\right)$ is a bounded sequence of strictly positive real numbers, $u=\left(u_{k}\right)$ is a sequence of positive real numbers, and $A=\left(a_{n k}\right)$ is an infinite matrix. Then the spaces $V_{\mathscr{F}}^{I}\left[\Delta_{v}^{m}, \lambda\right.$, $A, u, p,\|\cdot \ldots, \cdot\|]_{0}, V_{\mathscr{F}}^{I}\left[\Delta_{v}^{m}, \lambda, A, u, p,\|\cdot, \ldots, \cdot\|\right]$, and $V_{\mathscr{F}}^{I}\left[\Delta_{v}^{m}\right.$, $\lambda, A, u, p,\|\cdot, \ldots, \cdot\|]_{\infty}$ are linear spaces over the real field $\mathbb{R}$.

Proof. The proof of the theorem is easy so we omit it. space:

For the next result we shall define the following sequence

$$
\begin{aligned}
V_{\mathscr{F}} & {\left[\Delta_{v}^{m}, \lambda, A, u, p,\|\cdot, \ldots, \cdot\|\right]_{\infty} } \\
& =\left\{x=\left(x_{k}\right) \in S_{X}^{n}:\right.
\end{aligned}
$$

$$
\begin{aligned}
& \sup _{n} \frac{1}{\lambda_{n}} \sum_{k \in I_{n}} a_{n k}\left[f_{k}\left(\left\|u_{k} \Delta_{v}^{m} x_{k}-L, z_{1}, \ldots, z_{n-1}\right\|\right)\right]^{p_{k}} \\
& <\infty\} .
\end{aligned}
$$

Theorem 7. Let $\mathscr{F}=\left(f_{k}\right)$ be a sequence of modulus functions and $p=\left(p_{k}\right)$ a bounded sequence of strictly positive real numbers. Then the space $V_{\mathscr{F}}\left[\Delta_{v}^{m}, \lambda, A, u, p,\|\cdot, \ldots, \cdot\|\right]_{0}$ is a paranormed space with the paranorm:

$$
\begin{aligned}
& g(x) \\
& =\sup _{n}\left(\frac{1}{\lambda_{n}} \sum_{k \in I_{n}} a_{n k}\left[f_{k}\left(\left\|u_{k} \Delta_{v}^{m} x_{k}, z_{1}, \ldots, z_{n-1}\right\|\right)\right]^{p_{k}}\right)^{1 / G},
\end{aligned}
$$

where $G=\max (1, H)$ and $0<p_{k} \leq \sup _{k} p_{k}=H$.

Proof. Clearly $g(x)=g(-x)$ for $x \in V_{\mathscr{F}}^{I}\left[\Delta_{v}^{m}, \lambda, A, u, p\right.$, $\|\cdot, \ldots, \cdot\|]_{0}$. It is trivial that $u_{k} \Delta_{v}^{m} x_{k}=0$ for $x=0$. Since $f_{k}(0)=0$, we get $g(x)=0$ for $x=0$. Since $p_{k} / G \leq 1$, using Minkowski's inequality, we have

$$
\begin{aligned}
& \left(\frac{1}{\lambda_{n}} \sum_{k \in I_{n}} a_{n k}\left[f_{k}\left(\left\|u_{k} \Delta_{v}^{m}\left(x_{k}+y_{k}\right), z_{1}, \ldots, z_{n-1}\right\|\right)\right]^{p_{k}}\right)^{1 / G} \\
& \leq\left(\frac { 1 } { \lambda _ { n } } \sum _ { k \in I _ { n } } a _ { n k } \left[f _ { k } \left(\left\|u_{k} \Delta_{v}^{m} x_{k}, z_{1}, \ldots, z_{n-1}\right\|\right.\right.\right. \\
& \left.\left.\left.+\left\|u_{k} \Delta_{v}^{m} y_{k}, z_{1}, \ldots, z_{n-1}\right\|\right)\right]^{p_{k}}\right)^{1 / G} \\
& \leq\left(\frac{1}{\lambda_{n}} \sum_{k \in I_{n}} a_{n k}\left[f_{k}\left(\left\|u_{k} \Delta_{v}^{m} x_{k}, z_{1}, \ldots, z_{n-1}\right\|\right)\right]^{p_{k}}\right)^{1 / G} \\
& +\left(\frac{1}{\lambda_{n}} \sum_{k \in I_{n}} a_{n k}\left[f_{k}\left(\left\|u_{k} \Delta_{v}^{m} y_{k}, z_{1}, \ldots, z_{n-1}\right\|\right)\right]^{p_{k}}\right)^{1 / G} .
\end{aligned}
$$

Hence $g(x+y) \leq g(x)+g(y)$. Finally, to check the continuity of scalar multiplication, let $\alpha$ be any complex number; therefore by definition

$$
\begin{aligned}
& g(\alpha x) \\
& =\sup _{n}\left(\frac{1}{\lambda_{n}} \sum_{k \in I_{n}} a_{n k}\left[f_{k}\left(\left\|u_{k} \Delta_{v}^{m} \alpha x_{k}, z_{1}, \ldots, z_{n-1}\right\|\right)\right]^{p_{k}}\right)^{1 / G} \\
& \leq C_{\alpha}^{H / G} g(x),
\end{aligned}
$$


where $C_{\alpha}$ is a positive integer such that $\alpha \leq C_{\alpha}$. Let $\alpha \rightarrow 0$ for any fixed $x$ with $g(x) \neq 0$. By definition, for $|\alpha|<1$, we have

$$
\begin{array}{r}
\frac{1}{\lambda_{n}} \sum_{k \in I_{n}} a_{n k}\left[f_{k}\left(\left\|u_{k} \Delta_{v}^{m} \alpha x_{k}, z_{1}, \ldots, z_{n-1}\right\|\right)\right]^{p_{k}}<\epsilon, \\
\text { for } n>N(\epsilon) .
\end{array}
$$

Also, for $1 \leq n \leq N$, taking $\alpha$ small enough, since $f_{k}$ is coninuous, we have

$$
\frac{1}{\lambda_{n}} \sum_{k \in I_{n}} a_{n k}\left[f_{k}\left(\left\|u_{k} \Delta_{v}^{m} \alpha x_{k}, z_{1}, \ldots, z_{n-1}\right\|\right)\right]^{p_{k}}<\epsilon .
$$

Now (12) and (13) imply that $g(\alpha x) \rightarrow 0$ as $\alpha \rightarrow 0$. This completes the proof.

Theorem 8. Let $u=\left(u_{k}\right)$ be a sequence of positive real numbers. Then for $m \geq 1$ the following inclusions:

(i) $V_{\mathscr{F}}^{I}\left[\Delta_{v}^{m-1}, \lambda, A, u,\|\cdot, \ldots, \cdot\|\right]_{0} \quad c \quad V_{\mathscr{F}}^{I}\left[\Delta_{v}^{m}, \lambda, A, u\right.$, $\|\cdot, \ldots, \cdot\|]_{0}$

(ii) $V_{\mathscr{F}}^{I}\left[\Delta_{v}^{m-1}, \lambda, A, u,\|\cdot, \ldots, \cdot\|\right]_{\infty} \quad \subset \quad V_{\mathscr{F}}^{I}\left[\Delta_{v}^{m}, \lambda, A, u\right.$, $\|\cdot, \ldots, \cdot\|]_{\infty}$

are strict.

Proof. We will prove the result for $V_{\mathscr{F}}^{I}\left[\Delta_{v}^{m-1}, \lambda, A, u\right.$, $\|\cdot, \ldots, \cdot\|]_{0}$ only. The others can be proved similarly.

Suppose $x \in V_{\mathscr{F}}^{I}\left[\Delta_{v}^{m-1}, \lambda, A, u,\|\cdot, \ldots, \cdot\|\right]_{0}$; by definition, for every $\delta>0$ and $z_{1}, \ldots, z_{n-1} \in X$, we have

$$
\left\{n \in \mathbb{N}: \frac{1}{\lambda_{n}} \sum_{k \in I_{n}} a_{n k}\left[f_{k}\left(\left\|u_{k} \Delta_{v}^{m-1} x_{k}, z_{1}, \ldots, z_{n-1}\right\|\right)\right] \geq \delta\right\}
$$

$\in I$.

By the property of modulus function, we have

$$
\begin{aligned}
& \frac{1}{\lambda_{n}} \sum_{k \in I_{n}} a_{n k}\left[f_{k}\left(\left\|u_{k} \Delta_{v}^{m} x_{k}, z_{1}, \ldots, z_{n-1}\right\|\right)\right] \\
& \leq \frac{1}{\lambda_{n}} \sum_{k \in I_{n}} a_{n k}\left[f_{k}\left(\left\|u_{k} \Delta_{v}^{m-1} x_{k}, z_{1}, \ldots, z_{n-1}\right\|\right)\right. \\
& \left.+f_{k}\left(\left\|u_{k} \Delta_{v}^{m-1} x_{k+1}, z_{1}, \ldots, z_{n-1}\right\|\right)\right] \\
& \leq D \frac{1}{\lambda_{n}} \sum_{k \in I_{n}} a_{n k}\left[f_{k}\left(\left\|u_{k} \Delta_{v}^{m-1} x_{k}, z_{1}, \ldots, z_{n-1}\right\|\right)\right] \\
& +D \frac{1}{\lambda_{n}} \sum_{k \in I_{n}} a_{n k}\left[f_{k}\left(\left\|u_{k} \Delta_{v}^{m-1} x_{k+1}, z_{1}, \ldots, z_{n-1}\right\|\right)\right] \text { by (7). }
\end{aligned}
$$

Now, for given $\delta>0$, we have

$$
\begin{gathered}
\left\{n \in \mathbb{N}: \frac{1}{\lambda_{n}} \sum_{k \in I_{n}} a_{n k}\left[f_{k}\left(\left\|u_{k} \Delta_{v}^{m} x_{k}, z_{1}, \ldots, z_{n-1}\right\|\right)\right] \geq \delta\right\} \\
\subseteq\left\{n \in \mathbb{N}: \frac{1}{\lambda_{n}} \sum_{k \in I_{n}} a_{n k}\left[f_{k}\left(\left\|u_{k} \Delta_{v}^{m-1} x_{k}, z_{1}, \ldots, z_{n-1}\right\|\right)\right]\right. \\
\left.\geq \frac{\delta}{2 D}\right\} \\
\cup\left\{n \in \mathbb{N}: \frac{1}{\lambda_{n}} \sum_{k \in I_{n}} a_{n k}\left[f_{k}\left(\left\|u_{k} \Delta_{v}^{m-1} x_{k+1}, z_{1}, \ldots, z_{n-1}\right\|\right)\right]\right. \\
\left.\geq \frac{\delta}{2 D}\right\},
\end{gathered}
$$

for each $z_{1}, \ldots, z_{n-1} \in X$. Since $x \in V_{\mathscr{F}}^{I}\left[\Delta_{v}^{m-1}, \lambda, A, u\right.$, $\|\cdot, \ldots, \cdot\|]_{0}$, it follows that the sets on the right-hand side in the above containment belong to $I$. Hence $x \in V_{\mathscr{F}}^{I}\left[\Delta_{v}^{m-1}, \lambda, A\right.$, $u,\|\cdot, \ldots, \cdot\|]_{0}$. To show that the inclusion is strict, we give the following example.

We take $f_{k}(x)=x, \lambda_{n}=n$, and $A=\left(a_{n k}\right)=I$, for all $n, k \in \mathbb{N}, v=1$, and consider a sequence $x=\left(x_{k}\right)=k^{m-1}$; then $x \in V_{\mathscr{F}}^{I}\left[\Delta_{v}^{m}, \lambda, A, u,\|\cdot, \ldots, \cdot\|\right]_{0}$ but does not belong to $V_{\mathscr{F}}^{I}\left[\Delta_{v}^{m-1}, \lambda, A, u,\|\cdot, \ldots, \cdot\|\right]_{0}$ for $u=\left(u_{k}\right)=1, k \in \mathbb{N}$. This shows that the inclusion is strict.

Theorem 9. Let $\mathscr{F}^{\prime}=\left(f_{k}^{\prime}\right)$ and $\mathscr{F}^{\prime \prime}=\left(f_{k}^{\prime \prime}\right)$ be sequences of modulus functions. If $\limsup _{t \rightarrow \infty}\left(f_{k}^{\prime}(t) / f_{k}^{\prime \prime}(t)\right)=P>0$, then $V_{\mathscr{F}^{\prime}}^{I}\left[\Delta_{v}^{m}, \lambda, A, u, p,\|\cdot \ldots, \cdot\|\right] \subset V_{\mathscr{F}^{\prime \prime}}^{I}\left[\Delta_{v}^{m}, \lambda, A, u, p,\|\cdot, \ldots, \cdot\|\right]$.

Proof. Let $\lim \sup _{t \rightarrow \infty}\left(f_{k}^{\prime}(t) / f_{k}^{\prime \prime}(t)\right)=P$; then there exists a constant $M>0$ such that $f_{k}^{\prime}(t) \geq M f_{k}^{\prime \prime}(t)$ for all $t \geq 0$. Therefore, for each $z_{1}, \ldots, z_{n-1} \in X$, we have

$$
\begin{aligned}
& \frac{1}{\lambda_{n}} \sum_{k \in I_{n}} a_{n k}\left[f_{k}^{\prime}\left(\left\|u_{k} \Delta_{v}^{m} x_{k}-L, z_{1}, \ldots, z_{n-1}\right\|\right)\right]^{p_{k}} \\
& \geq(M)^{H} \frac{1}{\lambda_{n}} \sum_{k \in I_{n}} a_{n k}\left[f_{k}^{\prime \prime}\left(\left\|u_{k} \Delta_{v}^{m} x_{k}-L, z_{1}, \ldots, z_{n-1}\right\|\right)\right]^{p_{k}} .
\end{aligned}
$$

Then, for every $\delta>0$ and $z_{1}, \ldots, z_{n-1} \in X$, we have the following relationship:

$$
\left.\begin{array}{l}
\{n \in \mathbb{N}: \\
\quad \frac{1}{\lambda_{n}} \sum_{k \in I_{n}} a_{n k}\left[f_{k}^{\prime \prime}\left(\left\|u_{k} \Delta_{v}^{m} x_{k}-L, z_{1}, \ldots, z_{n-1}\right\|\right)\right]^{p_{k}} \geq \delta
\end{array}\right\}
$$




$$
\begin{aligned}
& \subseteq\{n \in \mathbb{N}: \\
& \quad \frac{1}{\lambda_{n}} \sum_{k \in I_{n}} a_{n k}\left[f_{k}^{\prime}\left(\left\|u_{k} \Delta_{v}^{m} x_{k}-L, z_{1}, \ldots, z_{n-1}\right\|\right)\right]^{p_{k}} \\
& \left.\geq \delta(M)^{H}\right\} .
\end{aligned}
$$

Since $x \in V_{\mathscr{F}^{\prime}}^{I}\left[\Delta_{v}^{m}, \lambda, A, u, p,\|\cdot, \ldots, \cdot\|\right]$, it follows that the set on left-side of the above containment belongs to $I$, which gives $x \in V_{\mathscr{F}^{\prime \prime}}^{I}\left[\Delta_{v}^{m}, \lambda, A, u, p,\|\cdot \ldots, \cdot\|\right]$.

Theorem 10. Let $\mathscr{F}=\left(f_{k}\right), \mathscr{F}^{\prime}=\left(f_{k}^{\prime}\right)$, and $\mathscr{F}^{\prime \prime}=\left(f_{k}^{\prime \prime}\right)$ be sequences of modulus functions. Then

(i) $V_{\mathscr{F}}^{I}\left[\Delta_{v}^{m}, \lambda, A, u, p,\|\cdot, \ldots, \cdot\|\right] \subset V_{\mathscr{F} \circ \mathscr{F}}^{I}\left[\Delta_{v}^{m}, \lambda, A, u, p\right.$, $\|\cdot, \ldots, \cdot\|]$,

(ii) $V_{\mathscr{F}^{\prime}}^{I}\left[\Delta_{v}^{m}, \lambda, A, u, p,\|\cdot, \ldots, \cdot\|\right] \cup V_{\mathscr{F}^{\prime \prime}}^{I}\left[\Delta_{v}^{m}, \lambda, A, u, p\right.$, $\|\cdot, \ldots, \cdot\|] \subset V_{\mathscr{F}^{\prime}+\mathscr{F}^{\prime \prime}}^{I}\left[\Delta_{v}^{m}, \lambda, A, u, p,\|\cdot, \ldots, \cdot\|\right]$.

Proof. (i) Let $x=\left(x_{k}\right) \in V_{\mathscr{F}^{\prime}}^{I}\left[\Delta_{v}^{m}, \lambda, A, u, p,\|\cdot, \ldots, \cdot\|\right]$, for every $\varepsilon>0$ and for some $L>0$ such that

$$
\left\{n \in \mathbb{N}: \frac{1}{\lambda_{n}} \sum_{k \in I_{n}} a_{n k}\left[f_{k}^{\prime}\left(\left\|u_{k} \Delta_{v}^{m} x_{k}-L, z_{1}, \ldots, z_{n-1}\right\|\right)\right]^{p_{k}} \geq \varepsilon\right\}
$$

$\in I$,

for each $z_{1}, \ldots, z_{n-1} \in X$. For given $\varepsilon>0$, we choose $\delta \in(0,1)$ such that $f_{k}(t)<\varepsilon$ for all $0<t<\delta$. On the other hand, we have

$$
\begin{aligned}
& \frac{1}{\lambda_{n}} \sum_{k \in I_{n}} a_{n k}\left[\left(f_{k} \circ f_{k}^{\prime}\right)\left(\left\|u_{k} \Delta_{v}^{m} x_{k}-L, z_{1}, \ldots, z_{n-1}\right\|\right)\right]^{p_{k}} \\
& =\frac{1}{\lambda_{n}} \sum_{k \in I_{n},\left[f_{k}^{\prime}\left(\left\|u_{k} \Delta_{v}^{m} x_{k}-L, z_{1}, \ldots, z_{n-1}\right\|\right)\right]^{p_{k}<\delta}} a_{n k}\left[\left(f_{k} \circ f_{k}^{\prime}\right)\right. \\
& \cdot\left(\| u_{k} \Delta_{v}^{m} x_{k}-L,\right. \\
& \left.\left.z_{1}, \ldots, z_{n-1} \|\right)\right]^{p_{k}} \\
& \sum_{\left.\lambda_{n \in I_{n},}, f_{k}^{\prime}\left(\left\|u_{k} \Delta_{v}^{m} x_{k}-L, z_{1}, \ldots, z_{n-1}\right\|\right)\right]^{p_{k} \geq \delta}} \begin{array}{c}
a_{n k} \\
\cdot\left[\left(f_{k} \circ f_{k}^{\prime}\right)\right. \\
\cdot\left(\| u_{k} \Delta_{v}^{m} x_{k}-L,\right. \\
\left.\left.z_{1}, \ldots, z_{n-1} \|\right)\right]^{p_{k}}
\end{array}
\end{aligned}
$$

$$
\begin{aligned}
\leq & (\varepsilon)^{H}+\max \left(1,\left(2 \frac{f_{k}(1)}{\delta}\right)^{H}\right) \frac{1}{\lambda_{n}} \\
& \cdot \sum_{k \in I_{n}} a_{n k}\left[f_{k}^{\prime}\left(\left\|u_{k} \Delta_{v}^{m} x_{k}-L, z_{1}, \ldots, z_{n-1}\right\|\right)\right]^{p_{k}},
\end{aligned}
$$

by Lemma 5 .

By using (19), we obtain $x \in V_{\mathscr{F}_{\circ} \mathscr{F}^{\prime}}^{I}\left[\Delta_{v}^{m}, \lambda, A, u, p\right.$, $\|\cdot, \ldots, \cdot\|]$.

(ii) The result of this part is proved by using the following inequality:

$$
\begin{aligned}
\frac{1}{\lambda_{n}} \sum_{k \in I_{n}} a_{n k}\left[\left(f_{k}^{\prime}+f_{k}^{\prime \prime}\right)\left(\left\|u_{k} \Delta_{v}^{m} x_{k}-L, z_{1}, \ldots, z_{n-1}\right\|\right)\right]^{p_{k}} \\
\leq \frac{D}{\lambda_{n}} \sum_{k \in I_{n}} a_{n k}\left[f_{k}^{\prime}\left(\left\|u_{k} \Delta_{v}^{m} x_{k}-L, z_{1}, \ldots, z_{n-1}\right\|\right)\right]^{p_{k}} \\
\quad+\frac{D}{\lambda_{n}} \sum_{k \in I_{n}} a_{n k}\left[f_{k}^{\prime \prime}\left(\left\|u_{k} \Delta_{v}^{m} x_{k}-L, z_{1}, \ldots, z_{n-1}\right\|\right)\right]^{p_{k}},
\end{aligned}
$$

where $\sup _{k} p_{k}=H$ and $D=\max \left(1,2^{H-1}\right)$.

Theorem 11. Let $\mathscr{F}=\left(f_{k}\right)$ be a sequence of modulus functions and $p=\left(p_{k}\right)$ a bounded sequence of strictly positive real numbers. Then $V^{I}\left[\Delta_{v}^{m}, \lambda, A, u, p,\|\cdot, \ldots, \cdot\|\right] \subseteq V_{\mathscr{F}}^{I}\left[\Delta_{v}^{m}, \lambda, A, u\right.$, $p,\|\cdot, \ldots, \cdot\|]$.

Proof. This can be proved by using the same techniques as in Theorem 10.

Theorem 12. Let $\mathscr{F}=\left(f_{k}\right)$ be a sequence of modulus functions. If $\lim \sup _{t \rightarrow \infty}\left(f_{k}(t) / t\right)=M>0$, then $V_{\mathscr{F}}^{I}\left[\Delta_{v}^{m}, \lambda, A, u\right.$, $p,\|\cdot, \ldots, \cdot\|] \subseteq V^{I}\left[\Delta_{v}^{m}, \lambda, A, u, p,\|\cdot, \ldots, \cdot\|\right]$.

Proof. Suppose $x=\left(x_{k}\right) \in V_{\mathscr{F}}^{I}\left[\Delta_{v}^{m}, \lambda, A, u, p,\|\cdot, \ldots, \cdot\|\right]$ and $\lim \sup _{t \rightarrow \infty}\left(f_{k}(t) / t\right)=M>0$, then there exists a constant $K>0$ such that $f_{k}(t) \geq K t$ for all $t \geq 0$. Thus, we have

$$
\begin{aligned}
& \frac{1}{\lambda_{n}} \sum_{k \in I_{n}} a_{n k}\left[f_{k}\left(\left\|u_{k} \Delta_{v}^{m} x_{k}-L, z_{1}, \ldots, z_{n-1}\right\|\right)\right]^{p_{k}} \\
& \quad \geq(K)^{H} \frac{1}{\lambda_{n}} \sum_{k \in I_{n}} a_{n k}\left[\left(\left\|u_{k} \Delta_{v}^{m} x_{k}-L, z_{1}, \ldots, z_{n-1}\right\|\right)\right]^{p_{k}},
\end{aligned}
$$

for each $z_{1}, \ldots, z_{n-1} \in X$. This completes the proof.

Theorem 13. If $0<p_{k} \leq q_{k}$ and $\left(q_{k} / p_{k}\right)$ be bounded. Then $V_{\mathscr{F}}^{I}\left[\Delta_{v}^{m}, \lambda, A, u, q,\|\cdot, \ldots, \cdot\|\right] \subset V_{\mathscr{F}}^{I}\left[\Delta_{v}^{m}, \lambda, A, u, p,\|\cdot, \ldots, \cdot\|\right]$.

Proof. It is easy to prove so we omit the detail.

\section{Statistical Convergence}

The notion of statistical convergence introduced by Fast [30] in 1951 and later developed by Fridy [31], Šalát [32] and many 
others. Furthermore, Kostyrko et al. [33] presented a very interesting generalization of statistical convergence called as $I$-convergence. Some recent developments in this regard can be found in [34-37] and many others.

In this section, we define a new class of generalized statistical convergent sequences with the help of an ideal, modulus functions and infinite matrix. We also made an effort to establish a strong connection between this convergence and the sequence space $V_{\mathscr{F}}^{I}\left[\Delta_{v}^{m}, \lambda, A, u, p,\|\cdot, \ldots, \cdot\|\right]$.

Definition 14. Let $I \subseteq P(\mathbb{N})$ be a non-trivial ideal and $\lambda=\left(\lambda_{n}\right)$ be a non-decreasing sequence. A sequence $x=\left(x_{k}\right) \in X$ is said to be $S_{\lambda}^{\Delta_{v}^{m}}(I, u, A,\|\cdot, \ldots, \cdot\|)$-convergent to a number $L$ provided that for every $\varepsilon>0, \delta>0$ and $z_{1}, \ldots, z_{n-1} \in X$, the set

$$
\begin{aligned}
\{n & \in \mathbb{N}: \frac{1}{\lambda_{n}}\left|\left\{k \in I_{n}: a_{n k}\left(\left\|u_{k} \Delta_{v}^{m} x_{k}-L, z_{1}, \ldots, z_{n-1}\right\|\right) \geq \varepsilon\right\}\right| \\
& \geq \delta\} \in I .
\end{aligned}
$$

In this case, we write $S_{\lambda}^{\Delta_{v}^{m}}(I, u, A)-\lim _{k \rightarrow \infty} \| u_{k} \Delta_{v}^{m} x_{k}, z_{1}, \ldots$, $z_{n-1}\|=\| L / \rho, z_{1}, \ldots, z_{n-1} \|$. Let $S_{\lambda}^{\Delta_{\nu}^{m}}(I, A, u,\|\cdot, \ldots, \cdot\|)$ denotes the set of all $S_{\lambda}^{\Delta_{\nu}^{m}}(I, u, A,\|\cdot, \ldots, \cdot\|)$-convergent sequences in $X$.

Theorem 15. Let $\mathscr{F}=\left(f_{k}\right)$ be a sequence of modulus functions and $0<\inf _{k} p_{k}=h \leq p_{k} \leq \sup _{k} p_{k}=H<\infty$. Then $V_{\mathscr{F}}^{I}\left[\Delta_{v}^{m}, \lambda, A, u, p,\|\cdot, \ldots, \cdot\|\right] \subset S_{\lambda}^{\Delta_{v}^{m}}[I, A, u,\|\cdot, \ldots, \cdot\|]$.

Proof. Suppose $x=\left(x_{k}\right) \in V_{\mathscr{F}}^{I}\left[\Delta_{v}^{m}, \lambda, A, u, p,\|\cdot, \ldots, \cdot\|\right]$ and $\varepsilon>0$ be given. Then for each $z_{1}, \ldots, z_{n-1} \in X$, we obtain

$$
\begin{aligned}
& \frac{1}{\lambda_{n}} \sum_{k \in I_{n}} a_{n k}\left[f_{k}\left(\left\|u_{k} \Delta_{v}^{m} x_{k}-L, z_{1}, \ldots, z_{n-1}\right\|\right)\right]^{p_{k}} \\
& =\frac{1}{\lambda_{n}} \sum_{k \in I_{n},\left\|u_{k} \Delta_{v}^{m} x_{k}-L, z_{1}, \ldots, z_{n-1}\right\| \geq \varepsilon} a_{n k}\left[f _ { k } \left(\| u_{k} \Delta_{v}^{m} x_{k}-L,\right.\right. \\
& \left.\left.\quad z_{1}, \ldots, z_{n-1} \|\right)\right]^{p_{k}} \\
& +\frac{1}{\lambda_{n}} \sum_{k \in I_{n},\left\|u_{k} \Delta_{v}^{m} x_{k}-L, z_{1}, \ldots, z_{n-1}\right\|<\varepsilon} a_{n k}\left[f _ { k } \left(\| u_{k} \Delta_{v}^{m} x_{k}-L,\right.\right. \\
& \frac{1}{\lambda_{n}} z_{k \in I_{n},\left\|u_{k} \Delta_{v}^{m}, \ldots, z_{n-1}-L, z_{1}, \ldots, z_{n-1}\right\| \geq \varepsilon} a_{n k}\left[f _ { k } \left(\| u_{k} \Delta_{v}^{m} x_{k}-L,\right.\right. \\
& \geq \frac{1}{\lambda_{n}} \sum_{k \in I_{n}}\left[f_{k}(\varepsilon)\right]^{p_{k}} \\
& \left.\left.z_{1}, \ldots, z_{n-1} \|\right)\right]^{p_{k}}
\end{aligned}
$$

$$
\begin{aligned}
& \geq \sum_{k \in I_{n}} \min \left(\left[f_{k}(\varepsilon)\right]^{h},\left[f_{k}(\varepsilon)\right]^{H}\right) \\
& \geq K \frac{1}{\lambda_{n}}\left|\left\{k \in I_{n}: a_{n k}\left(\left\|u_{k} \Delta_{v}^{m} x_{k}-L, z_{1}, \ldots, z_{n-1}\right\|\right) \geq \varepsilon\right\}\right|,
\end{aligned}
$$

where $K=\min \left(\left[f_{k}(\varepsilon)\right]^{h},\left[f_{k}(\varepsilon)\right]^{H}\right)$. Then for every $\delta>0$ and $z_{1}, \ldots, z_{n-1} \in X$, we have

$$
\begin{aligned}
& \left\{n \in \mathbb{N}: \frac{1}{\lambda_{n}}\left|\left\{k \in I_{n}: a_{n k}\left(\left\|u_{k} \Delta_{v}^{m} x_{k}-L, z_{1}, \ldots, z_{n-1}\right\|\right) \geq \varepsilon\right\}\right|\right. \\
& \geq \delta\} \\
& \subseteq\left\{n \in \mathbb{N}: \frac{1}{\lambda_{n}} \sum_{k \in I_{n}} a_{n k}\left[f_{k}\left(\left\|u_{k} \Delta_{v}^{m} x_{k}-L, z_{1}, \ldots, z_{n-1}\right\|\right)\right]^{p_{k}}\right. \\
& \geq K \delta\} .
\end{aligned}
$$

Since $x_{k} \rightarrow L\left(V_{\mathscr{F}}^{I}\left[\Delta_{v}^{m}, \lambda, A, u, p,\|\cdot, \ldots, \cdot\|\right]\right)$ so that $S_{\lambda}^{\Delta_{v}^{m}}(I, u$, A) $-\lim _{k \rightarrow \infty}\left\|u_{k} \Delta_{v}^{m} x_{k}, z_{1}, \ldots, z_{n-1}\right\|=\left\|L, z_{1}, \ldots, z_{n-1}\right\|$.

Theorem 16. Let $\mathscr{F}=\left(f_{k}\right)$ be a sequence of modulus functions and $p=\left(p_{k}\right)$ be a bounded sequence of strictly positive real numbers. If $0<\inf _{k} p_{k}=h \leq p_{k} \leq \sup _{k} p_{k}=H<\infty$ then $S_{\lambda}^{\Delta_{v}^{m}}[I, A, u,\|\cdot, \ldots, \cdot\|] \subset V_{\mathscr{F}}^{I}\left[\Delta_{v}^{m}, \lambda, A, u, p,\|\cdot, \ldots, \cdot\|\right]$.

Proof. By using ([11, Theorem 3.5]) we can prove easily.

Theorem 17. Let $\mathscr{F}=\left(f_{k}\right)$ be a bounded sequence of modulus functions and $p=\left(p_{k}\right)$ be a bounded sequence of strictly positive real numbers. If $0<\inf _{k} p_{k}=h \leq p_{k} \leq$ $\sup _{k} p_{k}=H<\infty$. Then $S_{\lambda}^{\Delta_{v}^{m}}[I, A, u,\|\cdot, \ldots, \cdot\|]=V_{\mathscr{F}}^{I}\left[\Delta_{v}^{m}, \lambda, A\right.$, $u, p,\|\cdot \ldots, \cdot\|]$ if and only if $\mathscr{F}=\left(f_{k}\right)$ is a bounded.

Proof. This part can be obtained by combining Theorems 15 and 16. Conversely suppose $\mathscr{F}=\left(f_{k}\right)$ be unbounded defined by $f_{k}(k)=k$ for all $k \in \mathbb{N}$. We take a fixed set $B \in I$, where $I$ is an admissible ideal and define $x=\left(x_{k}\right)$ as follows

$$
x_{k}= \begin{cases}k^{m+1}, & \text { for } n-\left[\sqrt{\lambda_{n}}\right]+1 \leq k \leq n, n \notin B \\ k^{m+1}, & \text { for } n-\left[\sqrt{\lambda_{n}}\right]+1 \leq k \leq n, n \in B \\ 0, & \text { otherwise. }\end{cases}
$$

For given $\varepsilon>0$ and for each $z_{1}, \ldots, z_{n-1} \in X$ we have,

$$
\begin{aligned}
& \lim _{n \rightarrow \infty} \frac{1}{\lambda_{n}}\left|\left\{k \in I_{n}: a_{n k}\left(\left\|u_{k} \Delta_{v}^{m} x_{k}-0, z_{1}, \ldots, z_{n-1}\right\|\right) \geq \varepsilon\right\}\right| \\
&<\frac{\left[\sqrt{\lambda_{n}}\right]}{\lambda_{n}} \longrightarrow 0,
\end{aligned}
$$


for $n \notin B$. Hence for $\delta>0$, there exists a positive integer $n_{0}$ such that $\left(1 / \lambda_{n}\right) \mid\left\{k \in I_{n}: a_{n k}\left\|u_{k} \Delta_{v}^{m} x_{k}-0, z_{1}, \ldots, z_{n-1}\right\| \geq\right.$ $\varepsilon\} \mid<\delta$ for $n \notin B$ and $n \geq n_{0}$. Now we have $\left(1 / \lambda_{n}\right) \mid\left\{k \in I_{n}\right.$ : $\left.a_{n k}\left\|u_{k} \Delta_{v}^{m} x_{k}-0, z_{1}, \ldots, z_{n-1}\right\| \geq \varepsilon\right\} \mid \geq \delta \subset\left\{B \cup\left(1,2, \ldots, n_{0}-\right.\right.$ $1)\}$. Since $I$ be an admissible ideal it follows that $S_{\lambda}^{\Delta_{v}^{m}}(I, u, A)-$ $\lim _{k \rightarrow \infty}\left\|u_{k} x_{k}, z_{1}, \ldots, z_{n-1}\right\| \rightarrow 0$ for each $z_{1}, \ldots, z_{n-1} \in X$.

On the other hand, if we take $p=\left(p_{k}\right)=1$, for all $k \in \mathbb{N}$ then $x_{k} \notin V_{\mathscr{F}}^{I}\left[\Delta_{v}^{m}, \lambda, A, u, p,\|\cdot, \ldots, \cdot\|\right]$. This contradicts the fact $S_{\lambda}^{\Delta_{v}^{m}}[I, A, u,\|\cdot, \ldots, \cdot\|]=V_{\mathscr{F}}^{I}\left[\Delta_{v}^{m}, \lambda, A, u, p,\|\cdot, \ldots, \cdot\|\right]$, so our supposition is wrong.

\section{Conflict of Interests}

The authors declare that there is no conflict of interests regarding the publication of this paper.

\section{Acknowledgments}

This project was funded by the Deanship of Scientific Research (DSR) at King Abdulaziz University, Jeddah, under grant no. (69-130-35-RG). The authors, therefore, acknowledge with thanks DSR technical and financial support.

\section{References}

[1] S. Gähler, "Lineare 2-normierte Räume," Mathematische Nachrichten, vol. 28, no. 1-2, pp. 1-43, 1964.

[2] A. Misiak, " $n$-inner product spaces," Mathematische Nachrichten, vol. 140, pp. 299-319, 1989.

[3] H. Gunawan, "On n-inner product, n-norms, and the CauchySchwartz inequality," Scientiae Mathematicae Japonicae, vol. 5, pp. 47-54, 2001.

[4] H. Gunawan, "The space of $p$-summable sequences and its natural n-norm," Bulletin of the Australian Mathematical Society, vol. 64, no. 1, pp. 137-147, 2001.

[5] H. Gunawan and M. Mashadi, "On n-normed spaces," International Journal of Mathematics and Mathematical Sciences, vol. 27, no. 10, pp. 631-639, 2001.

[6] K. Raj, S. K. Sharma, and A. K. Sharma, "Some difference sequence spaces in n-normed spaces defined by MusielakOrlicz function," Armenian Journal of Mathematics, vol. 3, pp. 127-141, 2010.

[7] A. Şahiner, M. Gürdal, S. Saltan, and H. Gunawan, "Ideal convergence in 2-normed spaces," Taiwanese Journal of Mathematics, vol. 11, no. 5, pp. 1477-1484, 2007.

[8] H. Kizmaz, "On certain sequence spaces," Canadian Mathematical Bulletin, vol. 24, no. 2, pp. 169-176, 1981.

[9] M. Et and R. Çolak, "On some generalized difference sequence spaces," Soochow Journal of Mathematics, vol. 21, no. 4, pp. 377386, 1995.

[10] Ç. A. Bektaş, M. Et, and R. Çolak, "Generalized difference sequence spaces and their dual spaces," Journal of Mathematical Analysis and Applications, vol. 292, no. 2, pp. 423-432, 2004.

[11] M. Et and A. Esi, "On Köthe-Toeplitz duals of generalized difference sequence spaces," Bulletin of the Malaysian Mathematical Sciences Society, vol. 23, no. 1, pp. 25-32, 2000.

[12] B. C. Tripathy and A. Esi, "A new type of difference sequence spaces," The International Journal of Science \& Technology, vol. 1, pp. 11-14, 2006.
[13] A. Esi, B. C. Tripathy, and B. Sarma, "On some new type generalized difference sequence spaces," Mathematica Slovaca, vol. 57, no. 5, pp. 475-482, 2007.

[14] B. C. Tripathy, A. Esi, and B. Tripathy, "On a new type of generalized difference Cesàro sequence spaces," Soochow Journal of Mathematics, vol. 31, no. 3, pp. 333-340, 2005.

[15] T. Bilgin, "Some new difference sequences spaces defined by an Orlicz function," Filomat, no. 17, pp. 1-8, 2003.

[16] M. Et, "Strongly almost summable difference sequences of order $m$ defined by a modulus," Studia Scientiarum Mathematicarum Hungarica, vol. 40, no. 4, pp. 463-476, 2003.

[17] S. A. Mohiuddine, K. Raj, and A. Alotaibi, "On some classes of double difference sequences of interval numbers," Abstract and Applied Analysis, vol. 2014, Article ID 516956, 8 pages, 2014.

[18] S. A. Mohiuddine, K. Raj, and A. Alotaibi, "Some paranormed double difference sequence spaces for Orlicz functions and bounded-regular matrices," Abstract and Applied Analysis, vol. 2014, Article ID 419064, 10 pages, 2014.

[19] S. A. Mohiuddine, K. Raj, and A. Alotaibi, "Generalized spaces of double sequences for Orlicz functions and bounded-regular matrices over $n$-normed spaces," Journal of Inequalities and Applications, vol. 2014, article 332, 2014.

[20] Y. Altin and M. Et, "Generalized difference sequence spaces defined by a modulus function in a locally convex space," Soochow Journal of Mathematics, vol. 31, no. 2, pp. 233-243, 2005.

[21] M. Mursaleen, " $\lambda$-statistical convergence," Mathematica Slovaca, vol. 50, pp. 111-115, 2000.

[22] E. Savaş and P. Das, "A generalized statistical convergence via ideals," Applied Mathematics Letters, vol. 24, no. 6, pp. 826-830, 2011.

[23] C. Belen and S. A. Mohiuddine, "Generalized weighted statistical convergence and application," Applied Mathematics and Computation, vol. 219, no. 18, pp. 9821-9826, 2013.

[24] P. Das, E. Savaş, and S. K. Ghosal, "On generalizations of certain summability methods using ideals," Applied Mathematics Letters, vol. 24, no. 9, pp. 1509-1514, 2011.

[25] M. Gürdal and H. Sarí, "Extremal $A$-statistical limit points via ideals," Journal of the Egyptian Mathematical Society, vol. 22, no. 1, pp. 55-58, 2014.

[26] M. Et, Y. Altin, and H. Altinok, "On some generalized difference sequence spaces defined by a modulus function," Filomat, no. 17, pp. 23-33, 2003.

[27] I. J. Maddox, "Sequence spaces defined by a modulus," Mathematical Proceedings of the Cambridge Philosophical Society, vol. 100, no. 1, pp. 161-166, 1986.

[28] E. Savaş and M. Mursaleen, "Matrix transformations in some sequence spaces," Istanbul Üniversitesi Fen Fakültesi Matematik Dergisi, vol. 52, pp. 1-5, 1993.

[29] S. Kumar, V. Kumar, and S. S. Bhatia, "Generalized sequence spaces in 2-normed spaces defined by ideal and a modulus function," Analele Ştiinţifice ale Universităţii, 2014.

[30] H. Fast, "Sur la convergence statistique," Colloquium Mathematicae, vol. 2, no. 3-4, pp. 241-244, 1951.

[31] J. A. Fridy, "On statistical convergence," Analysis, vol. 5, no. 4, pp. 301-313, 1985.

[32] T. Šalát, "On statistically convergent sequences of real numbers," Mathematica Slovaca, vol. 30, no. 2, pp. 139-150, 1980.

[33] P. Kostyrko, T. Salat, and W. Wilczynski, "I- convergence," Real Analysis Exchange, vol. 26, pp. 669-686, 2000. 
[34] M. Et, A. Alotaibi, and S. A. Mohiuddine, "On $\left(\Delta^{m}, I\right)$-statistical convergence of order $\alpha$," The Scientific World Journal, vol. 2014, Article ID 535419, 5 pages, 2014.

[35] F. Gezer and S. Karakuş, "I and I* convergent function sequences," Mathematical Communications, vol. 10, pp. 71-80, 2005.

[36] B. Hazarika and S. A. Mohiuddine, "Ideal convergence of random variables," Journal of Function Spaces and Applications, vol. 2013, Article ID 148249, 7 pages, 2013.

[37] M. Mursaleen and S. A. Mohiuddine, "On ideal convergence in probabilistic normed spaces," Mathematica Slovaca, vol. 62, no. 1, pp. 49-62, 2012. 


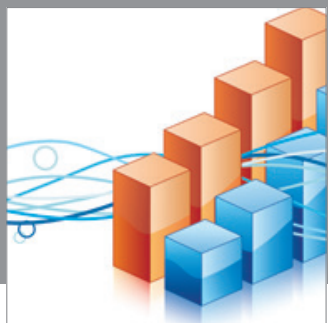

Advances in

Operations Research

mansans

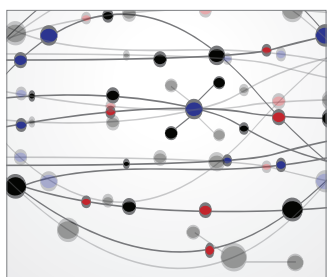

The Scientific World Journal
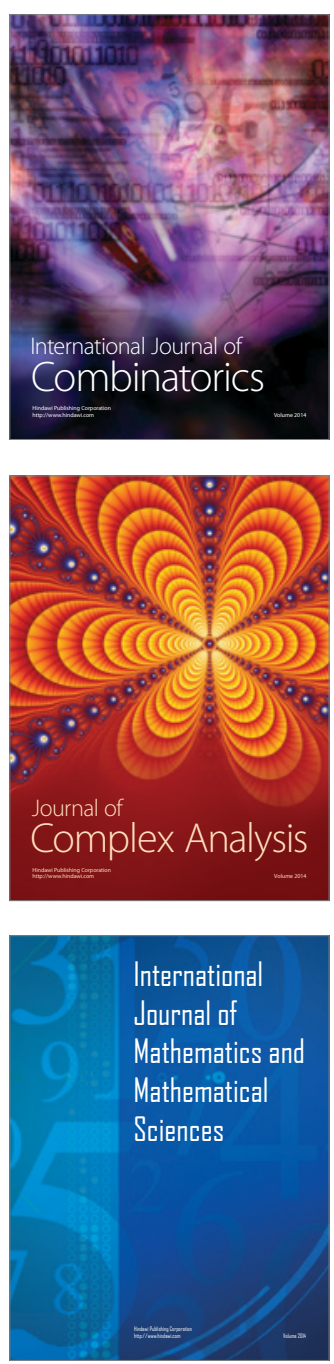
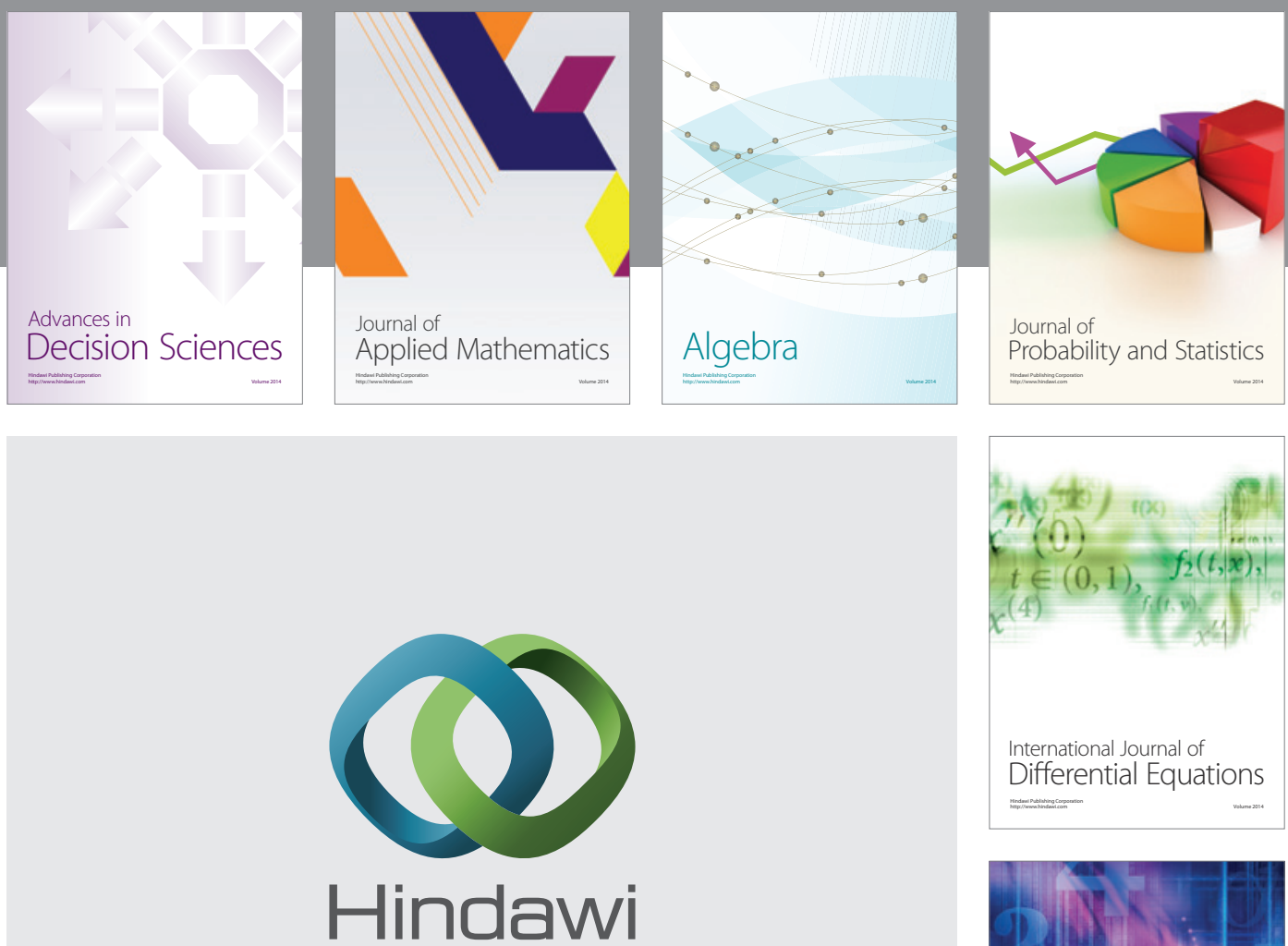

Submit your manuscripts at http://www.hindawi.com
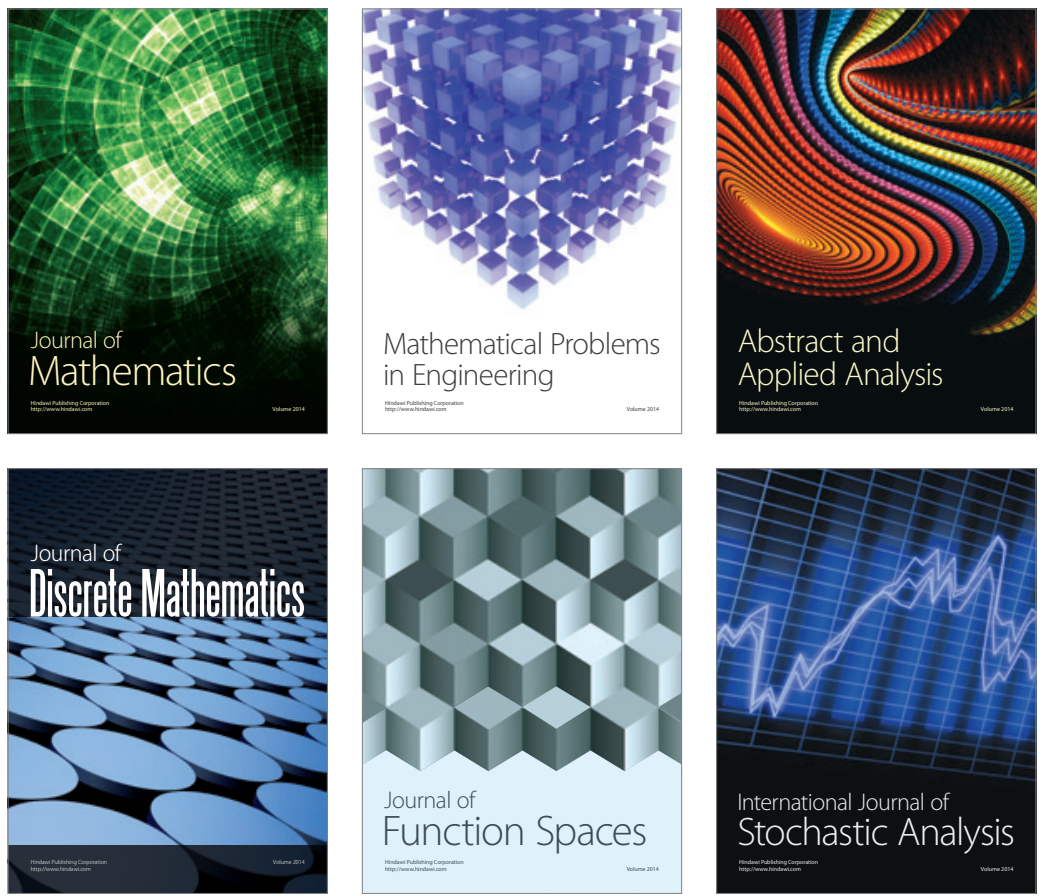

Journal of

Function Spaces

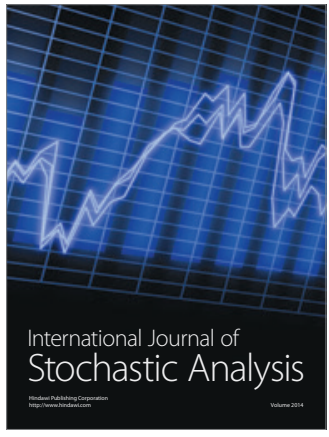

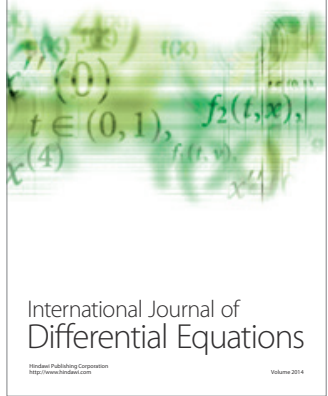
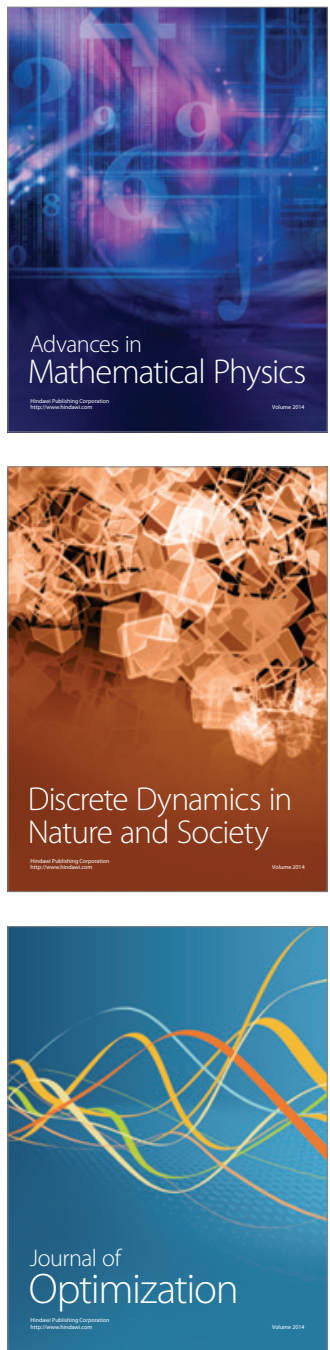